\title{
DISTRIBUTED BUSINESS PROCESS MANAGEMENT
}

\author{
Alexandra Augusta Pereira Klen, Ricardo J. Rabelo*, \\ Luiz Marcio Spinosa, Aureo Campos Ferreira \\ Federal University of Santa Catarina, Brazil
}

This chapter presents an effort to extend the intra-organizational logistics carried out by the classical PPC systems, with a higher level vision of the Virtual Enterprise's (VE) logistics, giving rise to an inter-organizational logistics. Getting, analyzing, making available and managing the information from and about $a V E$ as well as enabling the enterprises to make their logistics more efficiently, are the roles of the advanced coordination functionality called "logistics support" which has been implemented in the "Distributed Business Process Management System".

\section{A NEW WAY OF DOING BUSINESS}

The use of information technology is being encouraged increasingly for sharing and exchanging information among individuals and organizations in different places. This trend can be understood as a consequence of a new strategy of conducting business, which is the concept of Virtual Enterprises (VE). In the manufacturing sector, the Virtual enterprise is mostly composed of small and medium-sized enterprises acting as suppliers and having no definite relations, policies and implications. Hence, it is not difficult to perceive the degree of complexity in managing this kind of value-chain as well as coordinating the logistics of a business process that is distributed.

\section{Beyond structure: where are the boundaries of Business Processes?}

\section{Business Process}

A Business Process corresponds to a generic name to represent what an enterprise is going to do in a given business, regardless of its complexity, size and quantity. It can involve manufacturing processes, services, shipment, storage and many other activities.

\footnotetext{
- Federal University of Santa Catarina, Grucon - P.O. Box 476, Zip Code 88040 900, Florianopolis (SC) Brazil, Tel: + 55483319387 ext. 204, fax: + 5548234 1519, E-mail : rabelo@gsigma-grucon.ufsc.br 


\section{Distributed Business Process}

The VE must also deal with orders requests. In this case, these orders are known as distributed business processes (DBP). A DBP is a dynamic and temporary set of business processes (BP) which jointly gives rise to the end product of the VE. As the BPs are supposed to be performed by various enterprises, the enterprise that triggered the creation of a given VE normally coordinates its operation in order to avoid business chaos [Rabelo \& al., 1996]. In a VE scenario the management of the value-chain is a complex task, especially when some degrees of co-ordination are envisaged for support. Figure 1 illustrates a DBP concept via its production graph. The enterprise ' 2 ' (node 2) is currently the client-enterprise, whereas node-1, node-3 and node-5 are its direct suppliers. Each node has some (sub-)BPs under its responsibility (and so the respective Enterprise Activities -EAs), placed inside the dashed circle around them. They represent the value added by each enterprise on the production chain, in the course of time. For example, node-1 has to perform four interdependent BPs (BP1_10, BP1_22, BP1_22A and BP1_22B), whose result has to be sent to node-2 so that the BP1_1 can be carried out and so the whole production can be maintained.

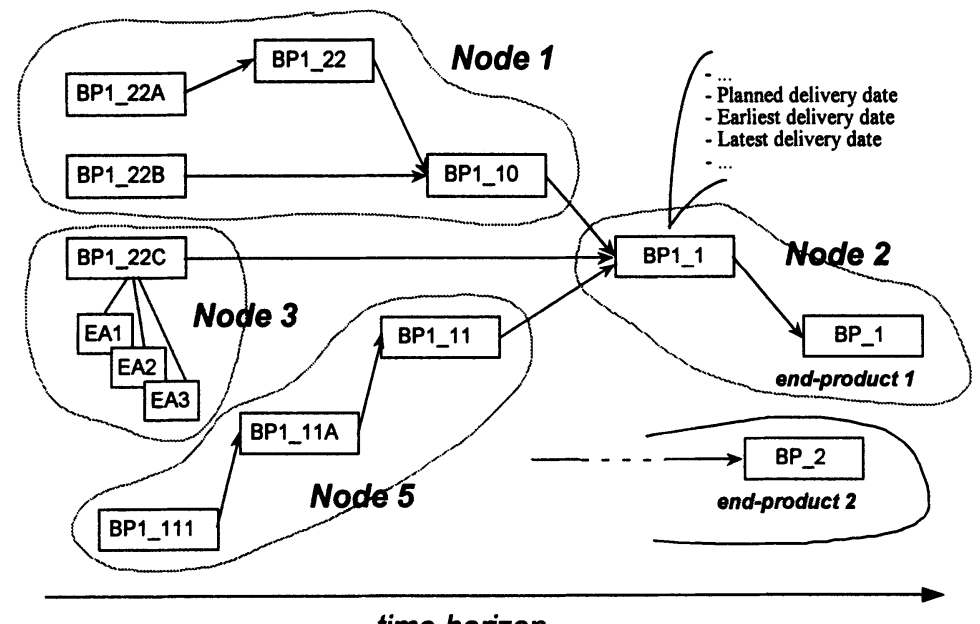

time horizon

Figure 1 - DBP concept

\section{Distributed Business Process Management - DBPM}

New logistics requirements, imposed by the virtual environment scenario, have brought about changes that have made logistics what it is today, seen as an integrated or embedded flow of material and information that has to be managed as one entity from the raw material to the final consumer. For this new perception of doing business, offered by the VE philosophy, the employment of Integrated Logistics (IL), as a concept meeting the needs of the distributed relations, may help understand its implications in the true integration of the VE. The Integrated Logistics framework focuses on overall performance instead of on individual performance, considering that the time is too short for multiple coordinating organizational levels and inventories to exist [Christopher, 1994] ${ }^{1}$. 
Furthermore, it is recognized that for the real benefits of the logistics concept to be implemented, there is a need to extend the logic of the logistics upstream to suppliers, and downstream to final customers. This is the concept of Supply Chain Management (SCM) [Leach \& al., 1996; Christopher, 1994].

The evolution of the logistics business concept has directly influenced the importance of improving understanding and managing the DBP. Since BPs are supposed to be performed by several enterprises (figure 1), the VE coordinator must, for instance, manage the operation of all BPs. As such, the VE is also liable to unexpected events, like BP delay, BP or DBP cancellation/modification, changes in DPB priorities, as well as to local communication deficiency and network overloading or failure. When one of these problems occurs and the enterprise responsible cannot solve the problem locally, it violates the DBP contract, and thereby causes a conflict. This conflict basically influences the DBP production dates (the planned delivery date) and can affect the whole production chain. Because the components of a DBP are all inter-related, an enterprise cannot (re)plan itself for its own benefits only, but it must also seek to benefit the whole net. Consequently, a close cooperation must exist both intra and inter-organizations in order to minimize the global loss in the "DBP contract".

The complexity of the resolution of a conflict may vary considerably. Because an enterprise usually plans with some temporal intervals between the BPs (just to absorb the unexpected occurrences), a re-planning negotiation may be relegated to adjust the planned delivery date either to the earliest delivery date or the latest delivery date. This policy could be one solution for a rapid contract re-negotiation and thus for a fast DBP re-establishment of plans. However, depending on the severity of the problem, the solution may involve a deeper - and possibly complex analysis. Many considerations and evaluations have to be taken into account, especially considering that an enterprise usually does not have just one DBP contracted, but a number of them, and this, in turn, can be indirectly affected by the problematic BP. Thus, the overall complexity makes it almost impossible for a user or a system to solve the problem individually. A single user lacks the knowledge and capabilities (in terms of time and technical background) and a system lacks the natural human 'business flexibility' and experience for good trade-off analysis / decisions. In this sense, a balanced approach seems to be a suitable solution to contemplate the enterprise with these two 'knowledge sources' simultaneously. In other words, there is a need for a Decision Support System (DSS) which can assist the user in decision-making. In this context, a DSS has to cope with the following stages [O'Neill,1995]: identifying the problem situation; acquiring and analyzing data; determining causes of problem; defining objectives; generating alternative solutions; comparing and evaluating alternative solutions; selecting (the 'best') solution.

A framework for this DSS must provide the decision-maker with selected information, describing both the competitive external environment and the way the enterprise is operating. In addition, it must also provide well-established decisionmaking support models and techniques.

In that sense, the DBPM system offers an enterprise with an environment that provides reliable and timely information about the supply-chain and a support for rapid decision-making facilitating, in this way, the coordination of the VE. 


\section{ADVANCED COORDINATION}

The co-ordination is a subject of great importance for the realization of VEs. Considering that the network of enterprises is formed due to the requirements of specific orders, there must be a way to co-ordinate these activities. In the PRODNET II project this is done through "Advanced Coordination Functionalities" (ACF). ACFs that correspond to specialized software modules being developed to help solve specific problems that require coordinated actions within the VE, improving the quality of supply-chain management.

The coordination functionalities are, in fact, a subset of the functional requirements for a VE. The PRODNET II architecture comprises a number of semiautonomous software modules that provides the enterprise with several other functionalities, such as: exchange of commercial data (using EDI/EDIFACT and Internet) - see chapter 6 - Part 2, exchange of technical product data (STEP) - see chapter 7 - Part 2, quality related information exchange - see chapter 8 - Part 2, VE information management - see chapter 3 - Part 2, the management of incomplete and imprecise orders - see chapter 4 - Part 2. All these "services" are supported by other basic software modules to guarantee the privacy and safety of communication, distributed data management, architecture/services configuration and global coordination among the modules [Camarinha-Matos \& al., 1998].

From the point of view of the enterprise, all those functionalities are seen as a "black boxes" which offer VE services - the PRODNET II Platform. It corresponds to an upper layer of software to be installed in the enterprise legacy systems (their internal modules) so that the enterprises can operate in a VE scenario in a "transparent" and integrated way. In fact, this platform comprises two main modules: the PRODNET Cooperation Layer (PCL) and the ACF modules (Figure 2).
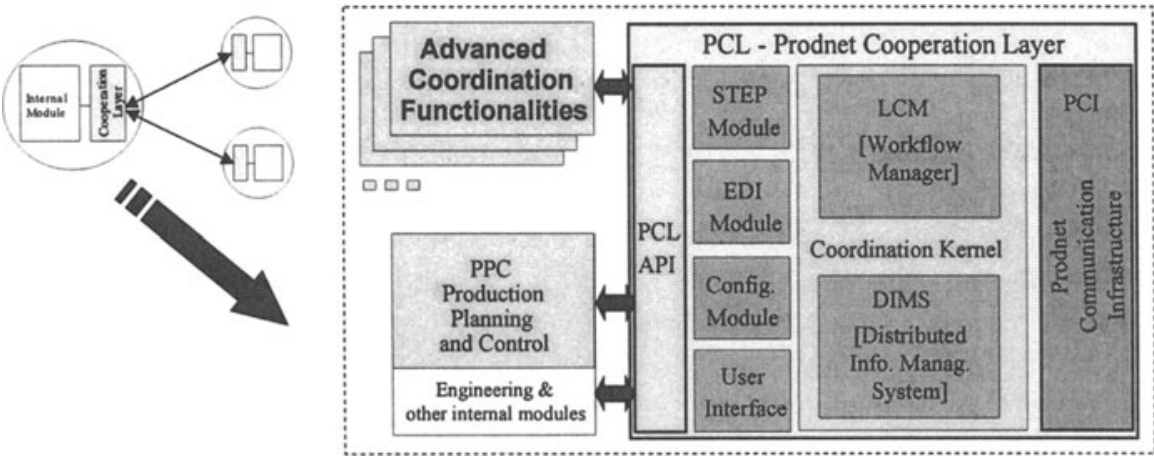

Figure 2 - The Advanced Coordination Functionalities in the PRODNET II Architecture

The ACFs can be seen as high-level services to be offered to an enterprise/user, who uses the basic services from the other PRODNET II Platform modules. The communication between the ACFs and the PCL is supported by means of a specific API (Application Program Interface), which was developed in the project. 


\section{Definition and Objective}

The Logistics Support is an ACF which seeks to provide means for obtaining, analyzing, making available and managing the information from and about a VE, enabling the enterprises to perform their logistics more efficiently. It was designed to act mainly in the operation phase of a VE life cycle [Spinosa \& al., 1998], although it may also be required in the dissolution phase as well as during the VE configuration i.e. in the creation phase.

In fact, the main fundamentals of the implemented functionalities reflect the consideration of the emerging concept of logistics called "integrated logistics management" (ILM). Within a wider logistics framework, ILM is focused on the operational level, whereas the Supply-Chain Management (SCM) is focused on the strategic level of the enterprise.

In this way, the ACF logistics support has been implemented in a system called "Distributed Business Process Management System" (DBPMS). The essential objectives of the DBPMS are to offer an enterprise with an environment that provides reliable and timely information about the supply-chain and a support for rapid decision-making, two extremely important enabling aspects to support the agility of the enterprise and hence its competitiveness.

The DBPMS extends the intra-organizational logistics carried out by the classical PPC systems, with a higher-level vision of the VE's logistics, giving rise to an inter-organizational logistics [Klen \& al., 1998].

\section{General Approach}

As mentioned before, the DBPMS is a kind of support system built up to help an enterprise for a better integrated supply-chain management, with an emphasis on the information flow. Its general approach is illustrated in the Figure 3.

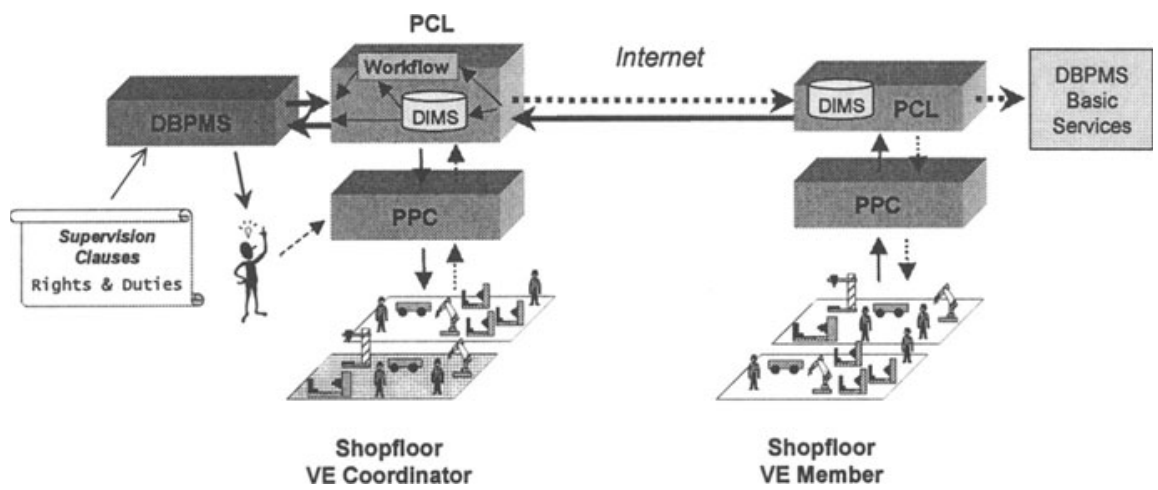

Figure 3 - DBPMS General Approach

The whole process starts when the VE-Coordinator wants to monitor, after a given VE is created, the processing of a particular BP at a given VE-Member's site. During the VE creation phase a set of agreements is made, including the 
specification of a number of information items that the VE-Member's PPC should send to the VE-Coordinator (like order status, remaining process time, the amount of parts already produced, etc.) as well as how often this should be done. These specifications are indicated in the "supervision clauses" (see next subsection), an information structure aggregated to the respective BP contract (the "purchase order contract" in the PPCs), which is filled up by the user via a specific graphic interface. Once a given VE is created, all the information about it - and that one required by the DBPMS - is stored in the Distributed Information Management System (DIMS) - see chapter 3 - Part 2 -, and fed by the VE-Coordinator's PPC - see chapter 8 Part 2.

Using the PRODNET II approach, the information being monitored (specified in the "rights" of the VE-Coordinator) is sent and managed by the DIMS module. This means that the VE-Member's PPC should obtain that information (in time cycles) from its shop floor and make it available in its DIMS so that the VE-Coordinator's DBPMS can have access to it in order to reason about it. Modifications in the VECoordinator's PPC may be carried out according to the decisions taken by the PPC (and not by the DBPMS). The continuous arrows in figure 3 illustrate this flow.

In another direction there are the rights of the VE-Member or, in other words, the duties of the VE-Coordinator. It is also important to provide the suppliers with reliable information. In this sense, the VE-Coordinator may have to inform the VEMember about a set of information, previously agreed on and specified in the supervision clauses / duties. The dashed arrows in figure 3 illustrate this flow. It means that a minimum set of DBPMS services should also be provided on the VEMember's side.

The inter-communication between the DIMS modules of the VE-Coordinator and the VE-Member is "transparently" supported by the DIMS' services as a federated and distributed database [Afsarmanesh \& al., 1998], which in turn involves, in the PRODNET II platform, a secure and encrypted information flow between the VE- Coordinator and the VE-Members [Osorio \& al., 1998].

\section{Main Functionalities}

The DBPMS functionalities implemented are comprised in three main blocks:

- VE Supervisor: One of the main problems the enterprises face nowadays is the difficulty in obtaining reliable information from the partners about the real status of some contracted BPs, which directly affect the DBP (the entire client order). This situation obliges the enterprises to re-plan their actions frequently due to the occurrence of unexpected events, representing delays or anticipations. It means an increase in the global costs of the enterprise because a delay or anticipation of one supplier can affect the execution of other inter-related BPs. This class seeks to offer an electronic means to get information from the suppliers so that the enterprises can constantly update their production plans and schedules [Rabelo \& al., 1997].

- Decision-Support for Logistics Management (DSS): The introduction of a decision-support system (DSS) that helps an enterprise to evaluate and to decide in the presence of a conflict is a key point for its efficient reaction. This kind of system can play its role much better if it is fed with reliable and real-time 
information from the supply-chain, which is supposed to be provided by the functionality previously explained. It can incorporate empirical knowledge from the more experienced users as well as analytical procedures as a support for its evaluations, including means for a trade-off analysis [Spinosa \& al., 1997]. Such a DSS assumes a strong interaction with the system user.

- Supervision Clause Configuration: It allows the user to interactively configure the supervision clauses to be applied to every VE-Member of a given VE. It comprises the mapping between the VE-Members' PPCs capabilities in terms of information gathering against the DBPMS needs, and also the set of (different) clauses at the levels of requested / purchase orders, required items and production orders.

Figure 4 illustrates the DBPMS architecture implemented, involving seven main modules:

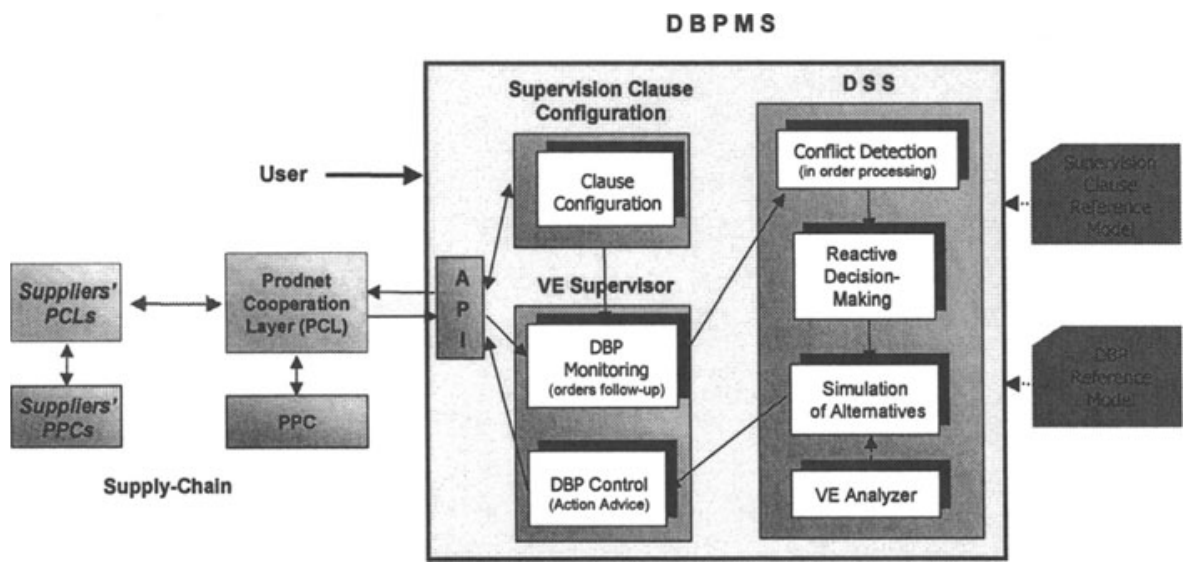

Figure 4 - DBPMS Architecture

- DBP monitoring: real-time information gathering from the suppliers' PPCs (in terms of orders);

- Conflict detection: detection and identification of unexpected problems during the orders processing;

- Reactive decision-making: support for decision-making according to the conflict detected;

- Simulation \& assessment of alternatives: support for the selection of a simulated decision by the user;

- DBP Control: set of actions that should be carried out in order to implement a selected decision.

- VE Analyzer: has the aim of providing an intraorganizational analysis of the VE in operation, as well as an interorganizational analysis of the alternative solutions. 
- Configuration: configuration of the rights and duties of the VE-Coordinator and VE-Members of a given VE.

Taking the main DBPMS modules into account, figure 5 illustrates its general control and information flow. All these modules will be further explained in the next, preceded by a description of the DBP as well as Supervision Clause Reference Models. Completing the frame, the integration and interoperation mechanism will also be highlighted.

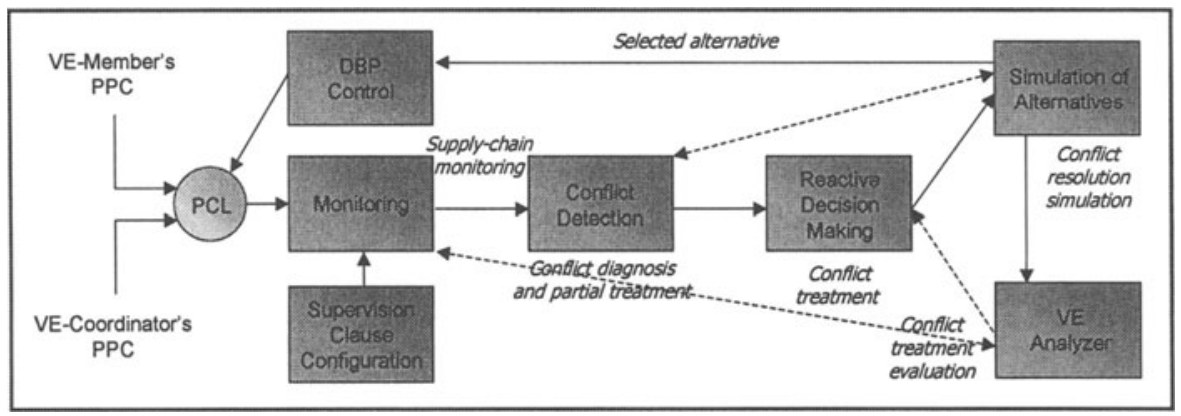

Figure 5-DBPMS general control and information flows

\section{DBP Reference Model}

For the system development, one of the important steps was the modeling of the Distributed Business Process (DBP). From the DBPMS point of view, a DBP is an object-oriented information structure that models the VE's goal, i.e. the "final product" in the PRODNET II context. In a real case, the DBP concept covers all the suppliers, all the orders and operations, as well as other information, involved in a given VE. It was designed in order to fulfil the DBPMS requirements in a VE scenario.

The DBP structure was designed taking some concepts of the CIM-OSA project into account [AMICE, 1993]. In this way, a DBP is a hierarchical model, composed of the following basic entities (figure 6).

There is an important observation related to the "Order" model. From the DBPMS point of view, this order refers to the so-called "production order", describing what a given supplier should do / produce within a DBP. In another instance, the EDI module and the PPC system view the "order model" in another perspective, classifying it as supplier order and customer order. This means that this order model to be stored / maintained in the DIMS module by the PPC system only specifies who the clients and the suppliers of the VE's supplier are for a given BP, but it does not identify (in the DIMS) the BP/order itself, as required by the DBPMS.

Regarding the usual lack of terminology standards in the production domain, the DBP modeling / specification was strongly based on the dictionary elaborated by the American Production \& Inventory Control Society [APICS, 1983].

\section{Supervision Clauses Model}

The existence of a "contract" in a VE scenario is just as real as it is in traditional relationships between two enterprises. In general, a contract specifies the rights and 
obligations from one enterprise to another, represented in terms of "clauses". This specification includes different kinds of clauses, comprising legal, technical and financial information.

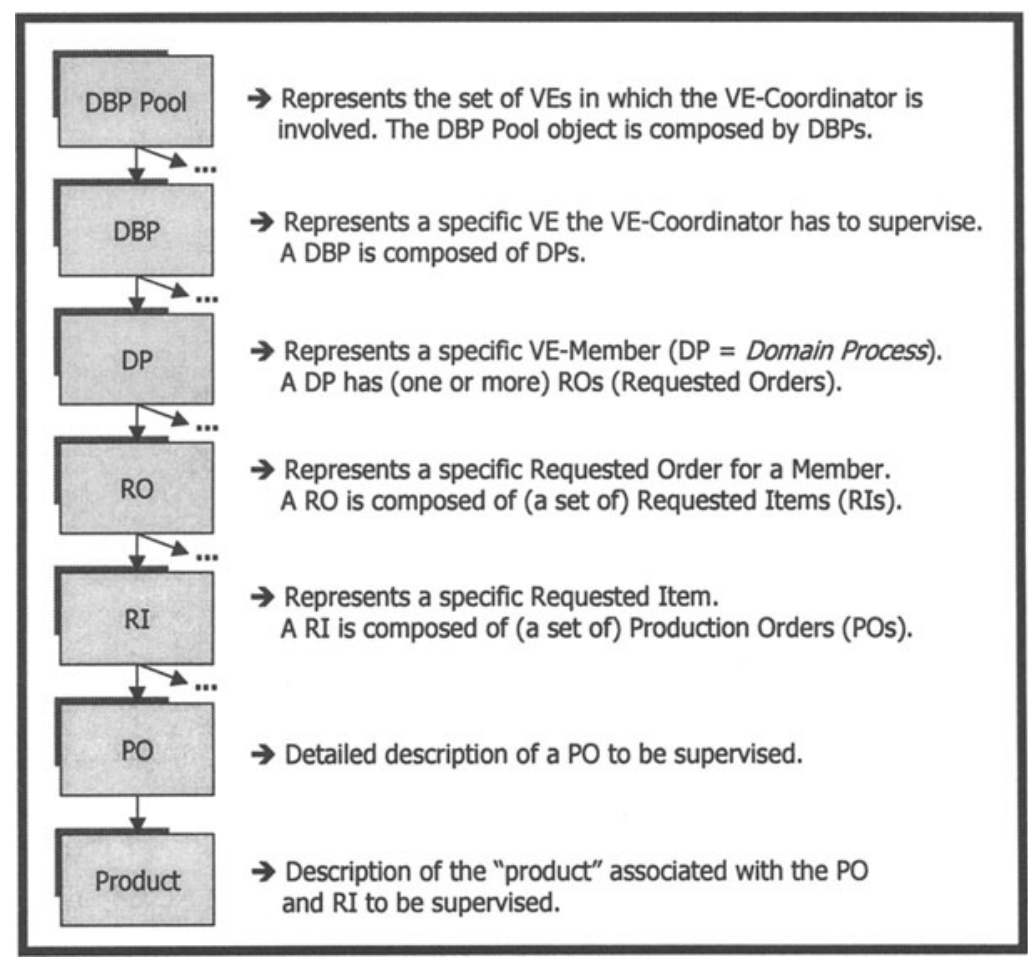

Figure 6 - DBP model general structure

However, from the supply-chain supervision point of view (i.e. for the DBPMS), an additional and innovative nature of clauses must exist: the "supervision clauses". Thus, supervision clauses are used to specify rights and obligations in terms of information access for monitoring purposes. In general, they specify what, how, when, where and how far a given set (within "classes") of information about an order from a given supplier should be monitored (this scenario was illustrated in figure 3).

The supervision clauses are an information model logically aggregated to the contract model and are necessary among the VE-Coordinator and Members. Figure 7 shows a generic contract structure and information content, positioning the supervision model / clauses.

\section{DBPMS Main Modules}

\section{Monitoring}

This module receives all the information to be monitored from both the VEMembers and the VE-Coordinator, which means that the DBPMS is prepared to deal with (a subset of) both intra and inter-organization conflicts. 


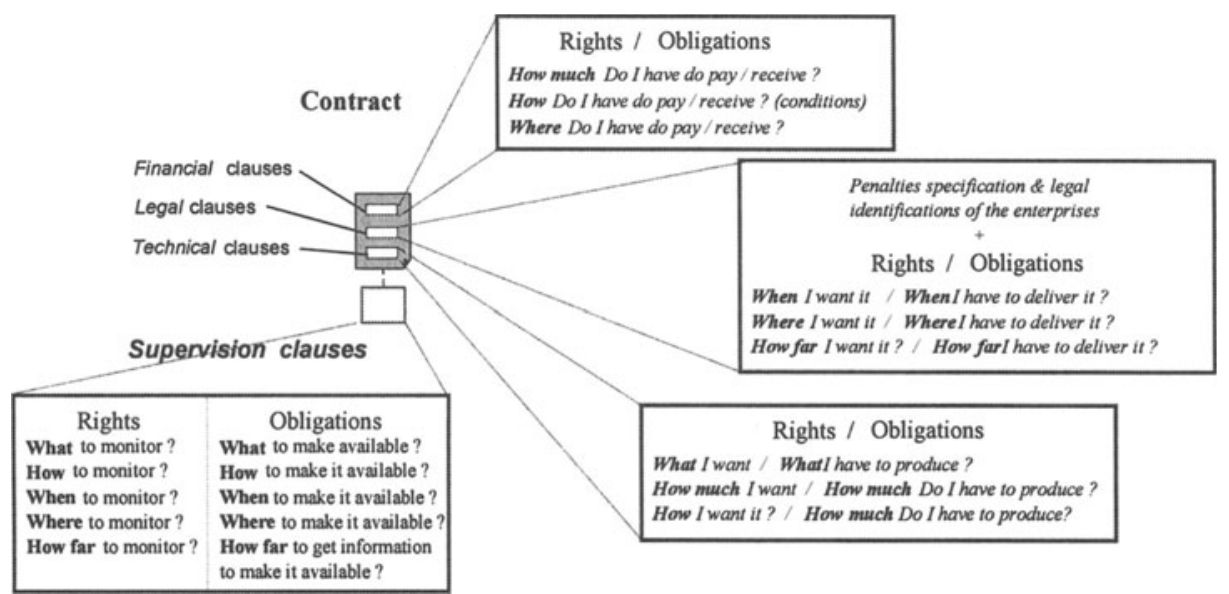

Figure 7 - Generic contract and supervision models

That information covers a given DBP, DP, BP, Order, product specification as well as the supervision clauses about them. From the DBPMS point of view, all the information comes from the PCL/DIMS module.

Once the information is received and organized into the DBPMS, it is passed on to the Conflict Detection module.

An example of one of the graphical user interfaces of this module is presented in figure 8, showing the main monitoring window.

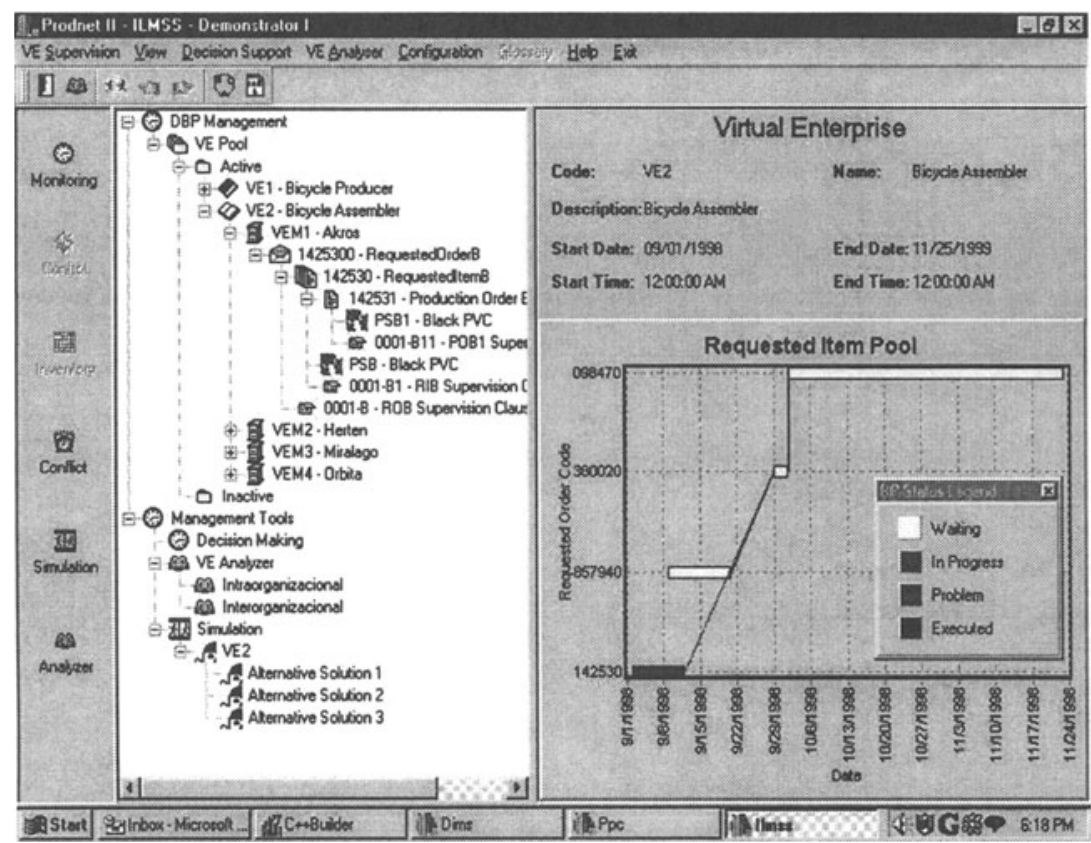

Figure 8 - Monitoring user interface 


\section{Conflict Detection}

This module receives the information that is being periodically monitored and then it checks whether it matches with what was planned, normally the order due dates and their execution status. If nothing wrong happens, the user keeps monitoring the order follow-up. In case a conflict is detected, it is identified and passed on to the Reactive Decision-Making module.

That checking is also carried out when the VE is created and the PPC puts all the DBP-related information in the DIMS, which in turn is accessed by the DBPMS. This action is useful in the sense that the PPC's user may fill in some order attribute wrongly without perceiving it. Thus, the DBPMS makes a full consistency check before instantiating its internal information models. When this kind of conflict is detected, the user is notified and called to confirm and respond accordingly.

An example of one of these graphical user interfaces is presented in figure 9, showing a conflict that has been detected.

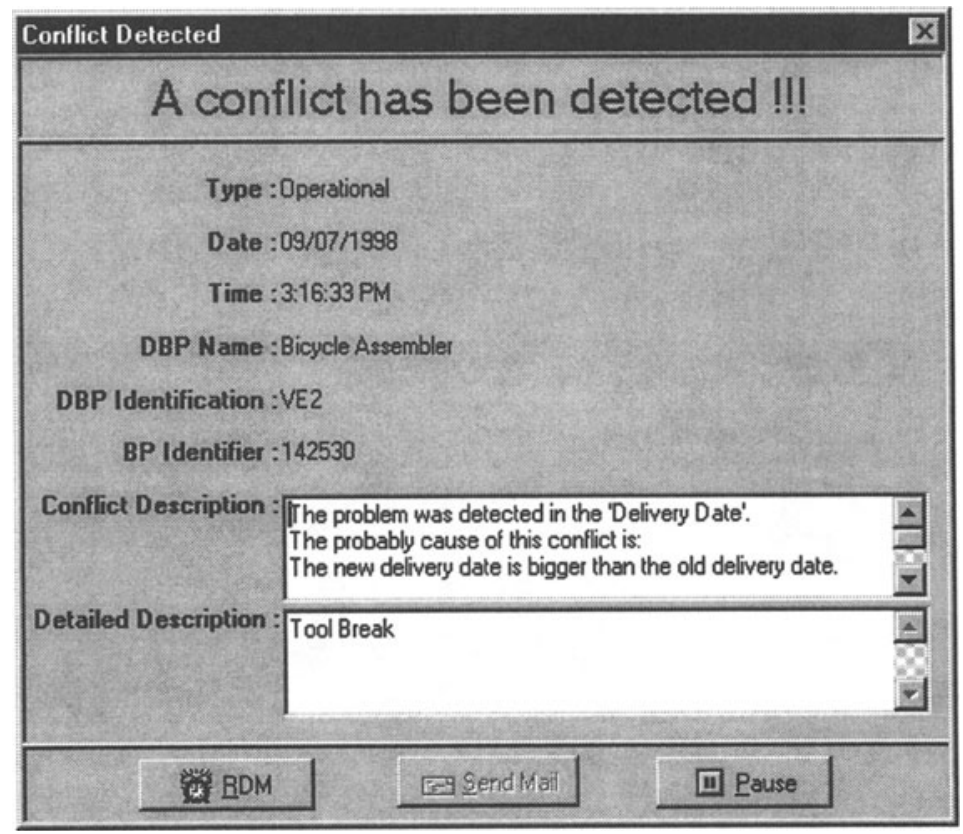

Figure 9 - Conflict Detection user interface

\section{Reactive Decision-Making}

This module receives the conflict identified by the Conflict Detection module and then tries to solve it. It is done by means of the application of a decision protocol, which was based on the case study of the two PRODNET II end users.

That protocol provides "solutions" that represent the set of actions that the (specialized end-user) enterprise uses to solve a conflict. An event picked up from the supply-chain is considered a conflict if it is not previously planned and/or if it affects the normal delivery dates of given BPs. It can be in terms of both order execution delay or anticipation in the order ending. 
That protocol involves several kinds of actions, such as interactive steps, sending recommendation messages to the suppliers, branches for new search and selection of partners, and rescheduling procedures with the evaluation of results.

If the conflicting supplier cannot internally solve a conflict, the VE coordinator should be notified. A conflict may affect only the BP under responsibility of that supplier (i.e. it does not affect the other BPs/suppliers) or, at worst, may affect other VE members. Considering that the prime VE goal is to finish its "product" on time, rescheduling actions are always triggered in order to (try to) keep the delivery date unaltered.

The DBPMS user has three possibilities for rescheduling: automatically, semiautomatically, and manually. In each case the rescheduling can be done in the DBPMS by means of the application of three of the most "popular" scheduling strategies: backward, forward and bottleneck, where a given BP is kept fixed and some of its neighbors are rescheduled backward and the others forward.

The Reactive Decision-Making module is the one responsible for doing the automatic rescheduling. Based on the conflict analysis and applying one of those three scheduling strategies, a "valid" (re)schedule is automatically generated. According to the protocol, if a valid schedule cannot be found, the DBPMS suggests a BP cancellation to the user.

Once a valid schedule is generated, it is passed to the Simulation of Alternatives module. In this module the user can try to improve its quality via either manual or semi-automatic rescheduling. In fact, in that previous case, when a valid schedule could not be generated, the Simulation of Alternatives module is also called so that the user can try to generate it by him/herself, based on his/her experience.

An example of one of the graphical user interfaces of this module is presented in figure 10 , showing the possibility offered to the user of choosing one or more rescheduling strategies.

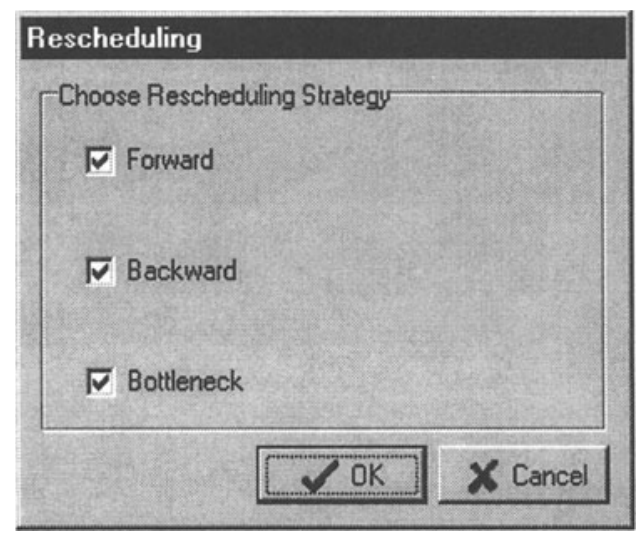

Figure 10 - Reactive Decision-Making user interface

\section{Simulation Alternatives}

This module receives a solution (a schedule) from the Reactive Decision-Making module so that the user can refine it and/or evaluate it directly. 
If the user understands that the "automatic" schedule is "good enough" to take a decision, he/she can set it immediately.

But, if the user considers that the suggested schedule can be improved, there is always the chance of changing it semi-automatically or starting the reschedule manually. In the manual way the user "sees" in a graphical interface a Gantt Diagram with the BPs involved and then he/she can work on it making manual changes. In order to avoid invalid specifications by the user, this module "calls" the Conflict Detection module to check them.

In the "semi-automatic" scheduling, the Simulation of Alternatives module automatically generates a schedule, which can be modified by the user. Moreover the user is also allowed to work on one of the new modified schedules (because they may be better than the previous ones). Then, the user can repeat this process on the "selected" schedule and new other schedules may be generated, until the user is certain that a given schedule is satisfactory enough for him/her.

An example of one of the graphical user interfaces of this module is presented in the figure 11 , showing when the user has to choose a rescheduling strategy.

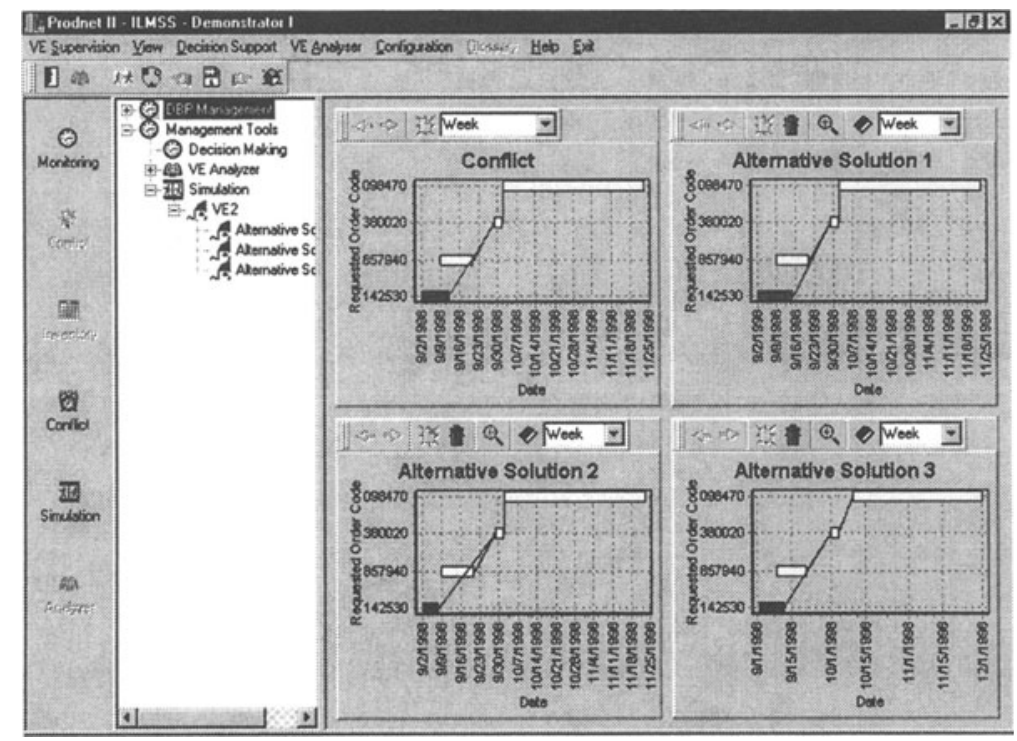

Figure 11 -Simulation of Alternatives user interface

\section{VE Analyzer}

This module is responsible for providing the user with the quality evaluation of a given schedule "sent" by the Simulation of Alternatives module, or of individual BPs from the VE, triggered by the Monitoring module.

The quality of a schedule is evaluated by means of the application of six production indicators (from the VE / inter-organization point of view), such as number of rescheduled BPs and DBP global extra-time. In addition to that, from the intra-organization point of view, the rescheduling consequences for each BP can be evaluated through fifteen BP indicators, such as due date, lead time and lateness. 
An example of one of the graphical user interfaces of this module is presented in figure 12, showing the intra-organization impact of a given schedule upon a "BP1".

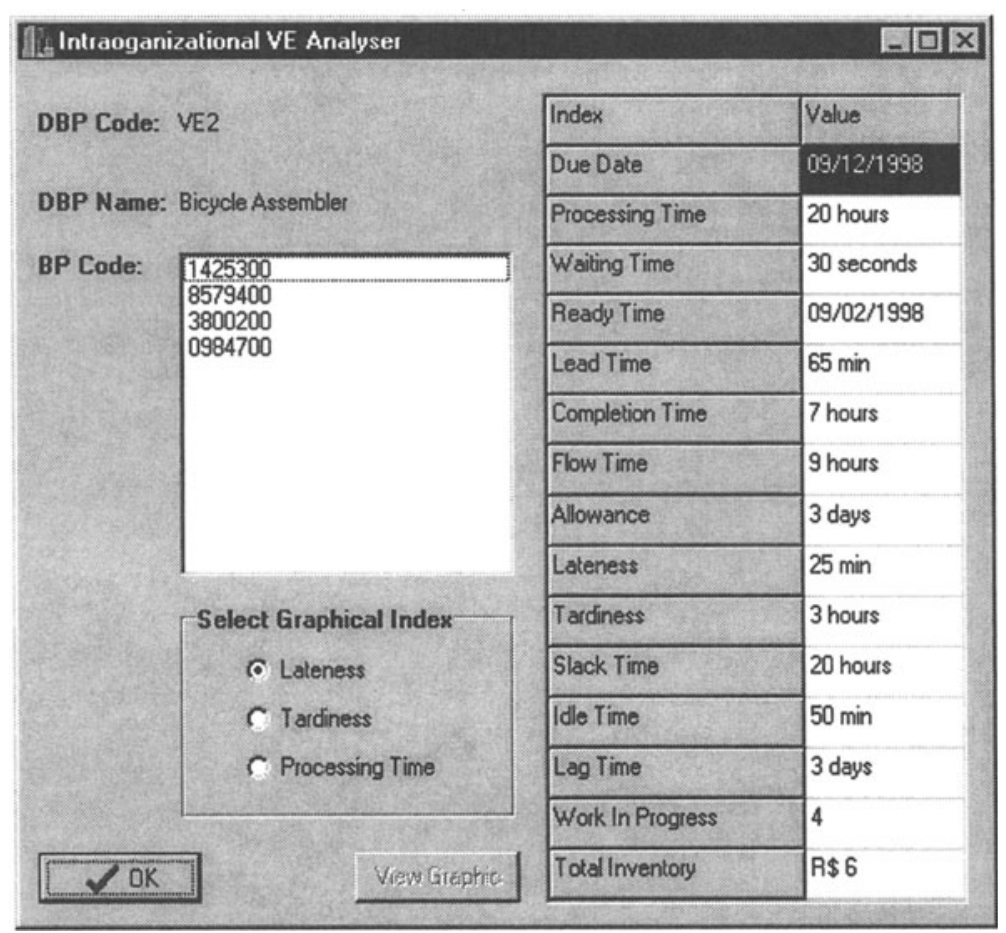

Figure $12-V E$ Analyzer user interface

\section{Control}

It corresponds to a set of actions and advice that should be carried out and be considered in order to "implement" a selected decision. It basically represents what has to be done once an alternative solution is chosen.

In the current DBPMS version, the user of the VE-Coordination site works with the DBPMS for decision-making support only. Based on the real data from the supply-chain obtained through the DBPMS monitoring module, he/she can simulate a number of decision alternatives for an eventual conflict. With this information he/she evaluates the alternatives and may feed its PPC with it. This means that there is not a direct/on-line integration between the decision simulated / "approved" and the PPC. Firstly this is because the DBPMS is a decision-support system, and not a decision-maker, secondly because a decision that can affect the whole supply-chain cannot be taken unilaterally, but rather after a global negotiation with the other enterprises involved in the modifications/rescheduling suggested by the DBPMS. Finally, because the DBPMS, as an ACF (i.e. acting at the VE level), cannot interfere in the "internal side" of the enterprise. 


\section{Supervision Clause Configuration}

In order to facilitate the user task in the specification of the supervision clauses for a given relation VE Coordinator / Member, a set of graphical interfaces was also developed.

From the DBPMS point of view, two enterprises cooperate with each other when they reach an agreement on which set of information one should be made available to the other about a BP under their responsibility, so as to allow a BP to be remotely monitored for supervision purposes. The enterprises are not obliged to cooperate in that sense, i.e. depending on the business profile and/or trust aspects, perhaps they may not cooperate. However, if they decide to cooperate, the DBPMS offers a supporting module for configuring the enterprise cooperation behavior.

The configuration of the supervision clauses is one of the first procedures the user should carry out once a VE is formed. He/she should specify which information the DBPMS should get from a member in time cycles. This information typically comprises production dates, quantities produced, order status, levels of information "visibility", decisions about sending auxiliary documentation in parallel or about sending monitoring information (production bulletins) in a text file to a client, etc.. An example of a configuration interface is shown in figure 13. The user should type some information and/or mark the options he/she is authorized to receive / provide, based on the previous general agreement.

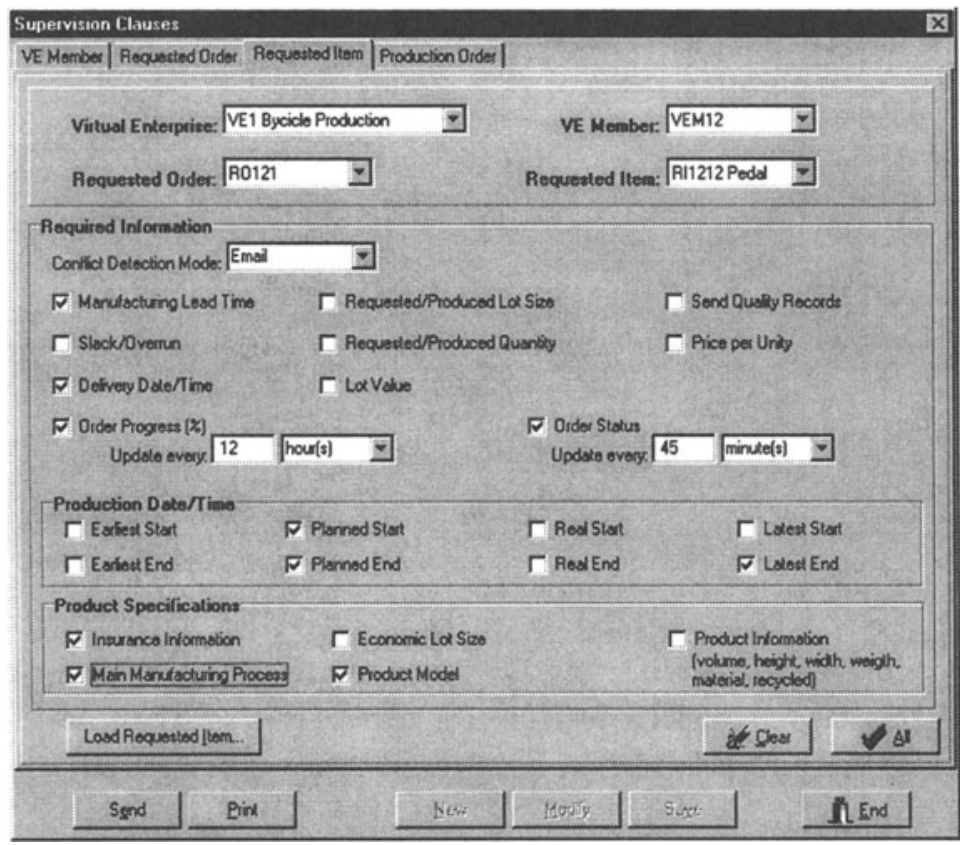

Figure 13 - Configuration for a given Requested Item

Although this configuration will normally take place only in the VE creation phase, it can also be modified during the VE operation. According to the evolution of business and changes on the agreements, the user can modify the previously 
configured clauses and set them accordingly. Both in the creation and operation phases, the DBPMS allows the user to store/recover supervision clauses according to the time from a "historic file".

Another aspect related to the supervision clause configuration concerns the capabilities of the PPCs of the VE-Members involved. In the DBPMS configuration module, when a VE is going to be configured, the user has to perform an interactive "mapping" between what a certain PPC is able to provide (in terms of "production information") and what it is not. Thus, the "users" (the VE-Coordinator and the respective VE-Member) do not need to waste much time negotiating "access right" levels if the PPC of the VE-Member is, for instance, too poor and hence only able to get little production information from its shop floor. This configuration step is only carried out if a given member is "new" for the coordinator (it has never worked with it before) or due to changes in the PPC of a previously known member (PPC capabilities, for instance).

Completing the frame, a special browser was developed to support the exception handling management in the DBPMS. This is very important for the tool interpretation and VE operation. Through this functionality the user can check the status of all interactions being carried out by the DBMPS and the other modules, by its internal modules and by the user. Moreover, it reduces the risk of crash also allowing the user some possibilities of intervention.

\section{Integration and Interoperation Mechanism}

The interoperation between DBPMS and PCL is necessary on two specific occasions. Initially, when the DBP Model - the main information structure of DBPMS - has to be instantiated and filled with information about the VE Members, as well as with the Orders information they have to execute. Later, during the Orders follow-up that involves gathering reliable information, interoperation is required again on three different levels: a generic level (concerning the status of the Requested Orders), an intermediary level (providing the status of the Items composing the Requested Orders) and a detailed level (concerning the Production Orders generated by the VE Member to execute each Item).

The level (s) that has (have) to be monitored is (are) specified by the VECoordinator in the Configuration Module.

To handle both moments, considering those different follow-up levels, two sets of interoperation services are provided according to the Common Interface Specification for PCL Modules Interoperation. Basically, the services request from PCL the necessary information involving either a Local Query Processing or a Federated Query Processing. The former involves mainly DIMS and the latter involves almost all PCL modules. In both cases, PCL answers the requests providing DBPMS with the "keys" to retrieve the information from DIMS, the main PCL data repository.

\section{CONCLUSIONS}

All the research in the literature and the conceptual work presented in this work were actually motivated by the need to identify some advanced co-ordination 
functionalities (ACFs) in order to try to avoid business chaos when coordinating one or more DBPs of a VE.

One of the ACFs identified is the Logistics Support, which sets out to support the means for obtaining, providing, and managing the information from and about a VE, enabling the enterprises to conduct their logistics more efficiently. Hence this ACF creates a system that incorporates part of the concepts from the Supply Chain Management and, mainly, from the Integrated Logistics Management. This system is known as Distributed Business Process Management System (DBPMS).

The DBPMS has the objective of provide an enterprise with an integrated environment. The system is modular, basically comprising three main interactive and cooperative modules: VE Supervisor (to monitor the DBP execution), DSS (Decision Support System, to help the user in the decision-making process based on the information received by the VE Supervisor), and Supervision Clause Configuration (where the rights and duties of the VE Coordinator and Members of a given VE are specified).

In sum, it can be said that the DBPMS main features are:

$\rightarrow$ Order follow-ups of the supply-chain, based on "supervision clauses" associated with the BPs' (orders) contracts;

$\rightarrow$ Interactive support for conflict analysis and resolution in the order processing, via reactive decisions;

$\rightarrow$ Configuration for each particular enterprise (way of operating);

$\rightarrow$ General support for Distributed Requirements Planning (DRP), since basic VE scheduling actions are provided;

$\rightarrow$ Integration with theProdnet Cooperation Layer (PCL):

- information integration: viaPCL's DIMS module;

- safety communication: viaPCL's PCI module;

- interoperation with thePCL's workflow engine module;

- interaction with the internal PPC: via PCL;

In addition, it is important to point out that considering:

- the DBPMS complexity,

- the innovative aspects that remain to be investigated, and

- the open questions still presented in the VE paradigm,

the experiments to be exploited by each DBPMS module had very limited scope and scenarios and were tested only in the PRODNET II project platforms. Furthermore, they were oriented to the demonstration pilot prototype, even because it is still not clear the possible overlapping between some PPC and DBPMS functionalities.

\section{Acknowledgements}

The authors would like to thank CNPq (The Brazilian Council for Research), project ProTeM-CC 680120/96-3 PRODNET II, and European Union, Esprit project 22647 PRODNET II for the financial support as well as all the project partners. A special thanks for the joint work also goes to the DBPMS development team of G-SIGMA, namely Mr. Allan Keller, Ms. Regina Bussolo, Mr. Rodrigo Ardigó and Mr. André Jacomino. 
${ }^{1}$ For further information about integrated logistics see also: Møller \& al., 1994; Slats et al., 1995; Christopher, 1992; and Bowersox and Closs, 1996.

\section{REFERENCES}

1. Afsarmanesh, H., Garita, C., Hertzberger, L.O. (1998), Virtual Enterprises and Federated Information Sharing, in Proceedings of the 9th IEEE International Conference on "Database and Expert Systems Applications", DEXA'98, Lecture Notes in Computer Science, Vienna, Austria.

2. AMICE (1993), CIMOSA: Open Systems Architecture for CIM, Springer-Verlag, Berlin.

3. APICS (1983), The Official Dictionary of Production and Inventory Management Terminology and Phrases, The American Prod. and Inventory Control Society, USA.

4. Bowersox, D. J. and Closs, D. J.; Logistical Management - The Integrated Supply Chain Process; McGraaw-Hill, 1996.

5. Camarinha-Matos, L.M., Afsarmanesh, H., Garita, C., Lima, C. (1998), Towards an architecture for virtual enterprises, Journal of Intelligent Manufacturing, Vol. 9, Issue 2.

6. Christopher, M.; New directions in Logistics, Logistics and Distribution Planning - strategies for management; ed. by James Cooper, Kogan Page Limited, $2^{\text {nd }}$ edition, 1994.

7. Klen, A. P.; Rabelo, R.J., Spinosa, L.M; Ferreira, A. C. (1998), Integrated Logistics in the Virtual Enterprise: The PRODNET II Approach, to be presented in IMS'98 - $5^{\text {th }}$ IFAC Workshop on Intelligent Manufacturing Systems, Gramado - Brazil.

8. Leach, N. P. et al.; Supply Chain Control: Trade-Offs and System Requirements; Proceedings of the ESPRIT - COPERNICUS Symposium, Budapest, Hungary, 12/1996.

9. Møller, C. et al.; Logistics Concept Development - A new Approach to Overall Integrated Logistics Modelling and Design, IFIP Working Group 5.7, Working Conference on Evaluation of production Management Methods, Brazil, 1994.

10. O'Neill, H. (1995), Decision Support in the Extended Enterprise, Ph.D. Thesis, Cranfield University., England.

11. Osorio, A. L.; Gibon, P.; Barata, M.(1998), Secure electronic commerce in virtual enterprises of SMEs, presented in Basys'98, IEEE IFIP International Conference on Balanced Automation System, Prague, Czech Republic, 24-26 August 1998. In Intelligent Systems for Manufacturing - MultiAgent Systems and Virtual Organizations, Eds. Luis M. Camarinha-Matos, Hamideh Afsarmanesh and Vladimir Marik, Kluwer Academic Publishers, pp. 207-218.

12. Rabelo, R. J.; Camarinha-Matos, L. M. (1996), Towards Agile Scheduling in Extended Enterprise, em "Balanced Automation Systems II - Implementation Challenges for Anthropocentric Manufacturing", Eds. Luis M. Camarinha-Matos and Hamideh Afsarmanesh, Chapman \& Hall. Proceedings BASYS'96 - IEEE / ECLA / IFIP - International Conference on Architectures and Design Methods for Balanced Automation Systems, Lisbon, Portugal.

13. Rabelo, R.J.; Spinosa, L. M. (1997), A Mobile-agent-based approach in supply-chain supervision in the food industry, Proceedings Agosoft'97 / Workshop on Supply-Chain Management, Vitoria, Brazil.

14. Slats, P. A., et al. (1995), Logistics Chain Modelling, European Journal of Operational Research No. $87,1-20$.

15. Spinosa, L. M.; Espinasse, B.; Chouraqui, E. (1997), For a Decision-Support System Model to Distributed Manufacturing Systems: a Multiagent and CIM-OSA based Approach, Proceedings of MCPL'97 - IFAC/IFIP Conference on Management and Control of Production and Logistics, Campinas (SP) Brazil, pp. 504-509.

16. Spinosa, L.M., Rabelo, R.J., Klen, A. P. (1998), High-Level Coordination of Business Processes in a Virtual Enterprise, in Proceedings of Prolamat'98 / The Tenth International IFIP TC5 WG-5.2 WG-5.3 Conference, Trento, Italy. 